\title{
Automate Minimization of Drive Tests for QoE Provisioning: The Case of Coverage Mapping
}

\author{
Vassilis Dalakas \\ Department of Informatics and Telematics \\ Harokopio University of Athens \\ 70 El. Venizelou, 17671 Athens, Greece
}

\begin{abstract}
The concept of Minimization of Drive Tests (MDT) has been developed in Third Generation Partnership Project (3GPP) specifications standard. With|MDT|Mobile Network Operators (MNOs) were enabled to remotely collect measurements indicating the network Quality of Service (QoS), as experienced by their users, correlated with actual location information. This results in wider application of use cases that allows network monitoring and optimization, without the need for conventional drive tests. To facilitate access to distributed geospatial data through a set of policies, common rules, and standards that would improve interoperability, this paper, proposes an open architecture, in compliance with the Open Geospatial Consortium (OGC) web services and the MDT architectures, enabling the automation of the MDT use cases and real-time service management.
\end{abstract}

\section{General Terms}

Service management, location-based services, geospatial web services

\section{Keywords}

Cellular radio coverage, quality of experience, quality of service

\section{INTRODUCTION}

Mobile Network Operators (MNOs) perform regular drive tests to assess the Quality of Service (QoS) of their networks, detect the coverage holes (a process called as coverage hole detection) and deploy a solution, which remedies/removes the coverage problem in the previously uncovered zones [8]. Unfortunately, drive tests tend to be limited on roads and other regions accessible by motor vehicles, and are not helpful in detecting coverage problems inside buildings not to mention that are an undesired source of pollution. Additionally, the use of drive tests has led to a huge amount of Operational Expenditure (OPEX), since it requires involvement of highly qualified technical staff and has delays in detecting problems on the network and in taking countermeasures [12].

Driven by these problems and demands, MNOs proposed to standardize solutions for remote sensing in Third Generation Partnership Project (3GPP) in order to reduce the effort and expense of drive testing. To this end the practical use cases of coverage, capacity, mobility optimization, parametrization of common channels and QoS verification, were considered in Release 9 [1]. Since then, the concept of Minimization of Drive Tests (MDT) has been enhanced and standardized for both Universal Mobile Telecommunications System (UMTS) and Long Term Evolution (LTE) in Release 11 specifications standard [2]. The 3GPP decided to continue its work based on an architecture involving Radio Access Network (RAN), to avoid redundancy and to enable close inter-working with the features of Self-Organizing Networks (SON) [14]. SON are dedicated to steadily adjusting the network's control parameters in order to configure the network to the greatest benefit of operators and users.

Apart from the possibilities of introducing new technologies to the network and to deploy more cells (e.g. small cells), the introduction of self-organization procedures is considered as a major opportunity to improve the network's performance in terms of QoS and Quality of Experience (QoE) |21|. However, QoE changes dynamically and depends on a large variety of factors [6]. With an effective use of MDT MNOs can potentially eliminate regular needs for conventional drive tests and realize real time service management of their networks [14]. Simulations have shown that by exploiting location specific network data obtained from MDT as an additional information input source for self-organization procedures, a significant performance gain is expected, since they can take their decisions upon an improved knowledge base [4]. While the concept of employing geo-statistical algorithms for the post processing of data, related with mobile signal receiving level, seems thoroughly examined [3, 16, 24 15], analysis and visualization of MDT data is not standardized [14].

On the other hand, the core business in Geographical Information Systems (GIS) is exactly this post processing, analysis and visualization of data containing geographical information. State-of-theart, GIS applications are based on a distributed model composed of independent and specialized geospatial web services, driven mainly by the need of offering distributed functionality over the Web [10]. These web services, that serve spatial information to users, have been evolved to an efficient way of interoperability and key pieces to build modular and distributed GIS applications. A major role to this evolution is played by the Open Geospatial Consortium (OGC), an international industry consortium consisted of companies, government agencies and universities that develops publicly available interface standards to support interoperable location-based solutions over the Web [22].

This paper focus on the automatic merging of MDT with concepts developed in OGC State-of-the-art geospatial web services are employed to facilitate service management by extracting added value 
information from the collection of specific Key Performance Indicators (KPIs), without dependence on conventional drive tests. Moreover, since QoS related data are collected from the users' side in real time, provisioning of $\mathrm{QoE}$ is feasible. To the best of our knowledge, this work is the only one exploiting web services to enhance the concept of MDT in a complete, standard and automatic manner. With the proposed approach, the MDT measurements provide the required input needed for the proposed services, and the use of geospatial techniques brings environment awareness to contemporary cellular networks as a step forward to reveal the actual QoE related with a certain geographical area. The key advantage of the architecture proposed here, is that it is not restricted to any specific operator, vendor, technology, equipment or algorithm, since it is compliant with both OGC and 3GPP standards.

As a proof of concept, a web-based prototype platform was implemented and the MDT use case of coverage [1] is examined. Automatic mapping and identification of real coverage holes, as well as coverage classification is presented, providing novel layers of valuable information to the RAN engineers. Coverage, without exaggeration, is the most important and the highest-priority target that has to be achieved by cellular operators. Without coverage provisioning, it is meaningless to talk about service, or QoS,QoEprovisioning. Therefore, cellular coverage prediction and enhancement remains a basic and prevailing area of research and investigation in wireless communications [8].

Implementation is based mainly on open source web components and technologies, similar to those presented in [15], leading to a feasible and low-cost solution based on the users' perspective. Please note that throughout this article, all the results presented are based on real data, collected from the field with users' equipment (UE) and not from drive tests, simulation or a planning tool, as it is common in the related literature.

This paper is structured as follows. An overview of the related work is presented in Section 2 while Section 3 presents the novel architecture. Section 4 deals with issues concerning the implementation and illustrates results. Finally conclusions are summarized in Section 5 .

\section{RELATED WORK}

3GPP standardized MDT functionality in order to reduce MNO $\mathrm{s}$ OPEX However, the benefit of MDT is not limited to OPEX reduction only. The geographical coverage of the testing is set by the presence and movement of real users, which is not coordinated, as opposed to the planned routes of conventional drive tests. The latter, reflects the real time QoE [14]. This location awareness is an essential value of the MDT standardization that cannot be achieved with conventional means. An MDT measurement is therefore likely to give a statistical view of network performance that is representative of how users sense the performance if treated appropriately [14]. To this end, measurement campaigns can be triggered. Such a campaign is likely to reach many indoor locations, including locations that would never be accessible by the operator's test personnel. Another benefit of MDT is that many different kinds of UE units can be used to collect information about normally rare events, making the MDT statistics a reasonable representation of user perceived statistics. This results in wider application of use cases that allows network service management and provisioning of QoE Recently, a data mining framework which allows to distinguish between satisfied and unsatisfied users in LTE for the MDT use case of QoS verification has been presented [6]. Data obtained from a LTE simulator were treated in a custom and non automatic manner to obtain results concerning throughput related QoE Although in

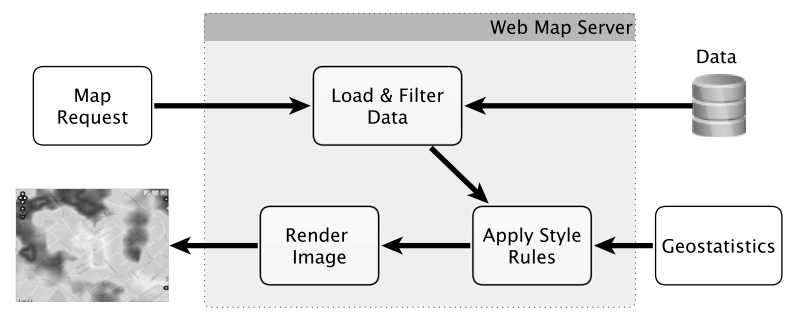

Fig. 1. Web Map Server.

the context of this paper focus is on the MDT use case of coverage, QoS verification could be considered in a future study, independent of the Radio Access Technology (RAT) since this approach can be easily adapted to adopt the same metrics with [6].

In the past, geospatial information calls for the concept of location/environment awareness were exploited in the context of Cognitive Radio [19]. Inspired by this connection, a cognitive tool namely, Radio Environment Maps (REMs) (developed as part of the research on cognitive wireless networks [25]) has been introduced and developed for diverse wireless network operation, management and optimization tasks [8] 9]. The authors, based on simulations, claim that with applying spatial interpolation techniques on the information coming from location-aware devices, REM could provide a realistic and remote representation of the ground-truth by automatically identifying the number, location and shape of the existing coverage holes [8]. This concept seems to fit into both the MDT and the Cognitive Radio framework [8], however, the obtained results were based only on models and input data from a planning tool. In other words, users' perspective was neglected since planning tools give only network's perspective. In addition, while geo-statistics has been used since early 1950?s in mining engineering field, and this theoretical framework has been successfully applied in a variety of spatial problems [18], even related with cellular networks [3, 16, 24 [15], the authors in [8] seem to ignore state-of-the-art on OGC web services [22].

Visualization of spatio-temporal data is an old issue, that has been the subject of several research papers [20, 13, 10, 17], even related with cellular networks [3, 16, 24, 15]. Existing tools for analyzing observations over time and geography are based on techniques and approaches from disciplines such as GIS geographical, scientific and information visualization. Moreover, for over a decade, OGC develops publicly available interface standards to support interoperable location-based solutions over the Web [23]. Geospatial web services have evolved to be an efficient way of interoperability and become key pieces to build modular and distributed GIS applications [10], supporting effectively the most common requirements and needs of spatial information users, such as discovering, accessing and visualization datasets. In addition, traditional discovery and visualization-based Spatial Data Infrastructures (SDI) is evolving to a more service-based vision in which geospatial web services are used not only to access geospatial data, but also to transform them and process them, often in service chains [10]. Most of these services implement interfaces defined by the OGC mainly with the help of a Web Map Server (see Fig. 11.

Examples of those services are Web Map Service (WMS) and Web Feature Service (WFS) which provide standard interfaces for querying and accessing map layers and geospatial data respectively [10]. However, these basic services seem to be insufficient to suit the processing requirements necessary for supporting operations related with MDT use cases. The Web Processing Services 


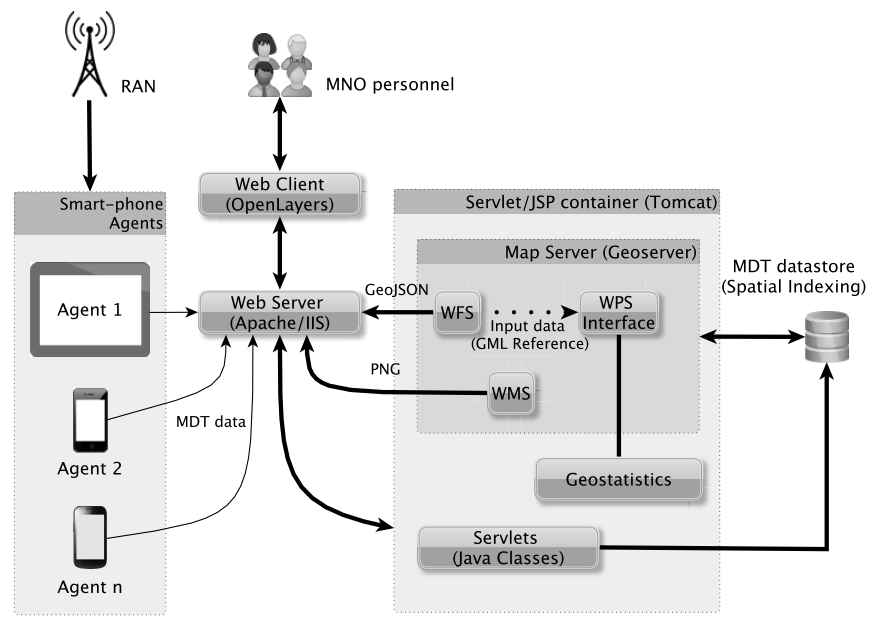

Fig. 2. Proposed architecture.

(WPS) specification provides interfaces for accessing more complex services and also for wrapping existing off-line services as web services [22]. This specification allows not only to discover or present spatial information but to provide geo-processing functionality, which are also independent of data and context, thereby providing high re-usability.

The capability of geo-statistical analysis in the context of mobile networks coverage prediction, simulation and tuning has been examined in [16]. Raw data, collected from drive tests, were exploited to evaluate different interpolation techniques in coverage prediction. Another application of geo-statistical modeling and interpolation, to the problem of radio environment mapping, was examined for the task of mapping the coverage and performance of a real WiMax network [24]. Recently, a web-GIS prototype has been proposed in [15] to identify problematic mobile signal areas via the web. Once again, various geo-statistical interpolation techniques were evaluated, this time with the help of proprietary GIS software, for the determination of the best method to predict mobile signal receiving level based on data from conventional drive tests. Proprietary equipment and software were employed for obtaining the drive tests' data that were imported manually to the database. However, none of the aforementioned efforts has proposed an architecture for the automatic post processing, analysis and visualization of MDT data based on standards. Not to mention real-time coverage mapping or real-time classification of QoE To address this important challenge, i.e., facilitating access to distributed geospatial data through a set of policies, common rules, and standards that would improve interoperability; this paper, proposes an open architecture, in compliance with the OGC web services and the MDT architectures that visualizes the users' perspective, instead of the common network's perspective.

\section{ARCHITECTURE}

Motivated by all these previously mentioned evolutions, an open architecture for cellular networks service management based on geospatial web services is proposed in Fig. 2 Since one cannot experiment with a real operating RAN of an existing MNO as indicated by the Control-plane (C-plane) MDT architecture followed by the 3GPP (see Fig. 3), the proposed architecture, is based, but it is not limited, on the User-plane (U-plane) MDT architecture [1]2]. By running a computationally lightweight application, com-

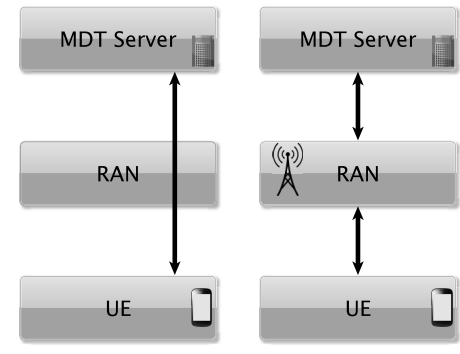

Fig. 3. U-plane MDT architecture (left) and C-plane MDT architecture (right).

mon smart-phones are transformed to MDT agents. Hence, network information is collected and transmitted over the Internet without involving RAN Centralized collection of these measurements is realized with a web service, responsible to process and store the data on a spatial database, imitating the role of the MDT server.

One could find some similarities of this MDT-driven part of the proposed architecture with the smart-phone based crowd-sourcing procedures discussed in [7]. Independently, of the adopted MDT architecture both approaches require the user's consent in order to collect data from its device. While motivating users to participate in such processes is of paramount importance, as success strongly depends on their volunteer contribution, such issues remains beyond the scope of this article. However, this architecture goes beyond the data collection process discussed in [7] since it deals with the automatic post-processing of the collected data and the meaningful visualization of the extracted features.

According to the proposed architecture, one has the ability to make spatio-temporal queries to the MDTdatabase via a web application. A user agent, commonly a web browser, initiates communication by making a request for a specific resource based on OGC web services and a web server, combined with a map server, responds with the content of that resource or an error message if unable to do so. The resource is typically an OGC map service output (i.e.,WFS, WMS and WPS. While the primary function of the web server is to serve content, a full implementation also includes ways of receiving content from clients. This feature is used when the smart-phone agents are sending MDT measurements.

This architecture has been implemented and tested, as a proof of concept, by automatically revealing real coverage holes, based on measurements of the received signal level. Specific implementation details will be presented in the following section as well as the results from geo-processing services that encapsulate all required tasks to access, analyze, and transform MDT data. However, OGC WPS services go beyond providing unique geo-processing services because it can be an interesting specification with which to wrap both spatial and non-spatial processing services, leading to increased interoperability between OGC Web Services Phase 9 (OWS) services and general purpose web services.

\section{IMPLEMENTATION AND RESULTS}

\subsection{Spatial Interpolation Method}

Please note that the proposed architecture realizes a multi sensor environment. Hence, data fusion techniques [5] could be employed with the aim of fusing and aggregating data from different sensors such as those collected through smart phones. Spatial interpolation methods might combine geospatial data "from multiple sensors and related information from associated databases to achieve improved 


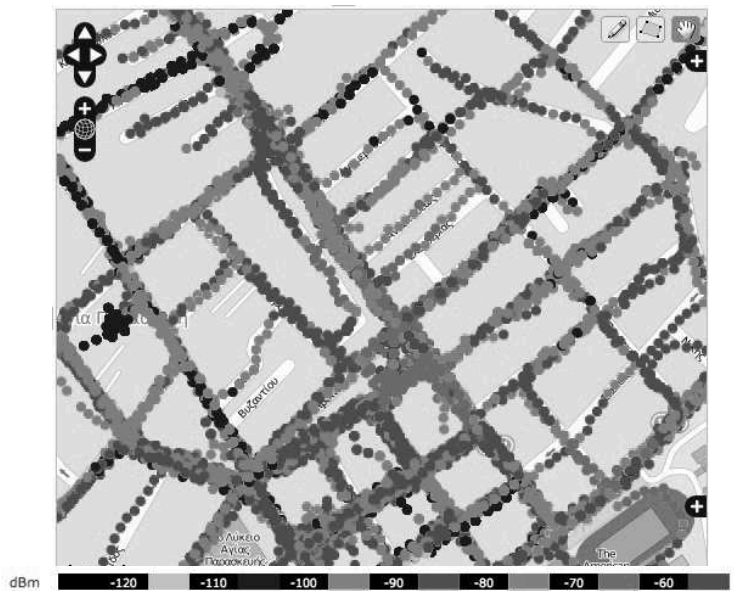

Fig. 4. Collected measurements depicted via WMS

accuracy and more specific inferences than could be achieved by the use of a single sensor alone", as stated by one of the well-known definition of data fusion by Hall and Llinas [11].

The obtained results were extracted with the Inverse Distance Weighted (IDW) method since it was recently found to outperform other popular spatial interpolation methods for the case of mobile signal levels [15]. Hence, a Vector-to-Raster transformation which computes an interpolated surface across a set of irregular observation points such as the MDT datasets was implemented as a WPS process. IDW interpolates sparse observations from a set of measurements to obtain a gridded dataset (surface). It is based on the assumption that in any given location $i$ the value of the field $f$ is best approximated by a combination of the $N$ closest observations weighted by a function of the inverse of the distance $r_{i n}$ between the $i$-th point and $n$-th observation [17]:

$$
f\left(\mathbf{r}_{i}\right)=\sum_{n=0}^{N} w\left(r_{i n}\right) f_{o}\left(\mathbf{r}_{n}\right)
$$

where $f_{o}\left(\mathbf{r}_{n}\right)$, is the observed value for each point. The algorithm operates on a regular grid of cells covering a specified extent in the input data space. The value of a cell, $f\left(\mathbf{r}_{i}\right)$, is determined by its proximity to the input observation points, using a summation of a-priori known functions (weights), $w\left(r_{i n}\right)$, for each observation point, $n$, defined as:

$$
w\left(r_{i n}\right)=-\left(\frac{1}{r_{i n}^{3}}\right) \quad 1 \leq n \leq N .
$$

\subsection{Mapping MDT data}

Fig. 4 illustrates 55734 measurements collected, classified and coloured according to their $\mathrm{dBm}$ level for the specific geographic area. The data consist of $3 \mathrm{G}$ received pilot powers, i.e., Received Signal Code Power (RSCP) values. Here, an urban area in the north east of Athens has been cosidered. For legal issues the name of the considered MNO is not revealed. Note that the base map presented here is imported from OpenStreet Maps.

The OGC|Styled Layer Descriptor (SLD) Profile of the OGCWMS encoding standard defines an encoding that extends the WMS standard to allow user-defined symbolization and colouring of geographic feature and coverage data. SLD addresses the need for users

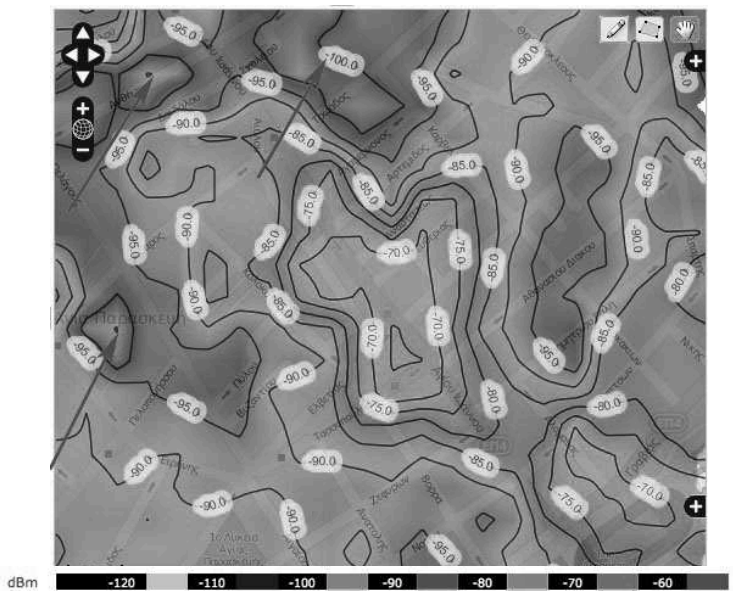

Fig. 5. Coverage map estimated via WPS and user experience classification depicted with iso-pleth lines separated on intervals of $5 \mathrm{dBms}$. These lines can be used to identify problematic areas for QoE (pointed out with arrows).

and software to be able to control the visual portrayal of the geospatial data. The ability to define styling rules requires a styling language that the client and server can both understand. The OGC Symbology Encoding (SE) standard provides this language, while the SLD profile of WMS enables application of SE to WMS layers using extensions of WMS operations. Additionally, SLD defines an operation for standardized access to legend symbols [22].

WMS and SLD are also employed to generate the coverage map illustrated in Fig. 5 The same measurements (i.e., vector data in GIS terminology) are spatially interpolated to create a surface (i.e., raster data in GIS terminology) representing an estimation of the experienced coverage. The same colormap is followed as in Fig 4 Moreover, in order to classify the users experience, another GIS technique has been applied. The vectorization of the just obtained raster map for the estimation of zones with RSCP values in intervals of $5 \mathrm{dBms}$ via a WPS tailor-made in Python. Hence, for Fig. 5 except the WMS protocol the WPS protocol is also exploited. Zones with values lower than $-95 \mathrm{dBms}$, identify coverages holes and are pointed out in Fig 5 with arrows for clarity. Please note that one is able to obtain similar maps of user experience for every collected KPI in the database. An excerpt of the SLD file style.sld written to support illustration purposes is given.

<?xml version="1.0" encoding="ISO-8859-1"?>

$<$ StyledLayerDescriptor version="1.0.0" ...> $<$ NamedLayer $>$

$<$ Name $>$ my_style $</$ Name $>$

<UserStyle>

$<$ Name $>$ my_style $</$ Name $>$

$<$ Title $>$ IDW style</Title $>$

$<$ FeatureTypeStyle>

$<$ Transformation>

<ogc:Function name="gs:VDSurface">

.

$</$ ogc: Function $>$

$</$ Transformation $>$

$<$ Rule $>$

$<$ Name $>$ rule $1</$ Name $>$

$<$ Title>Give Color to Raster</Title>

<Abstract>A raster with $100 \%$ opacity</Abstract> 


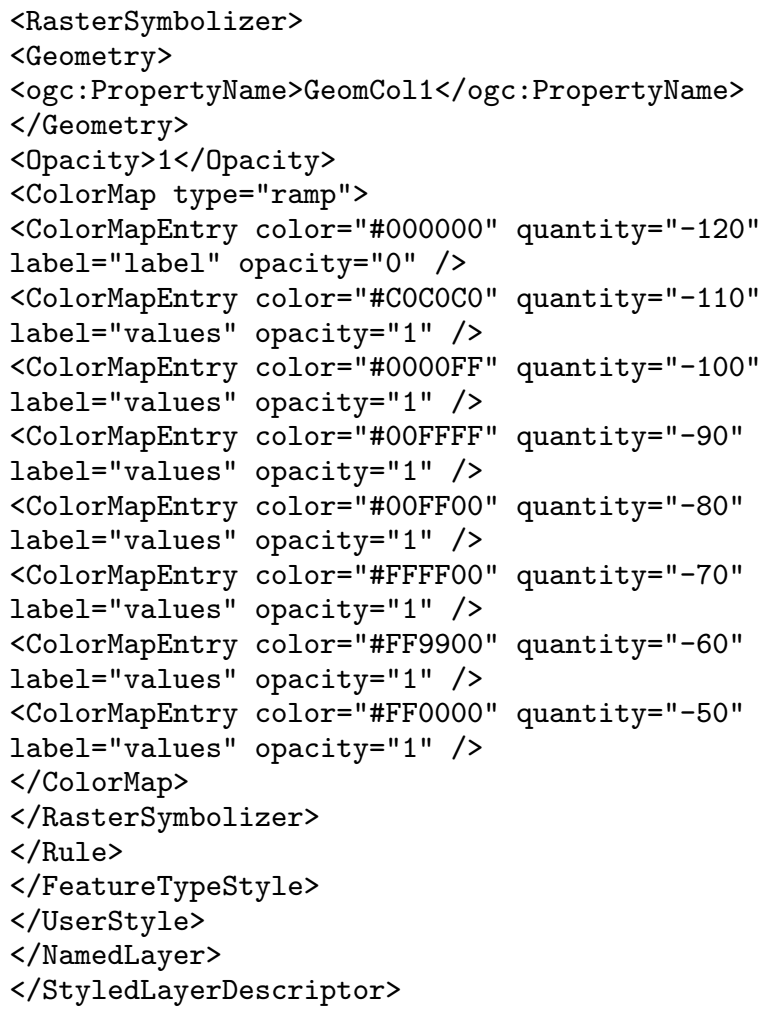

\section{CONCLUSIONS}

This paper presented an approach that enhances the MDT concept via standardized geospatial web services to automatically post process, analyse and visualise network specific KPIs For a proof of concept, a web-based prototype platform compliant with these standards was implemented and the automatic visualization of real coverage as well as classification of experience and identification of coverage holes, has been illustrated. Please note that all these are obtained in real-time. Focus is not on the geo-statistical techniques, already proposed as appropriate for the domain, but on the application of concepts and standards proposed by OGC and 3GPP towards service management in a feasible and automatic manner. The key advantage of the proposed architecture is that it is not restricted to any specific operator, vendor, technology, equipment or algorithm, since it is compliant with both OGC and 3GPP standards. Although the presented results are for the MDT use case of $3 \mathrm{G}$ coverage this proposal is not limited to that as long as there are more KPIs leading to a feasible and low-cost expandable solution for network service management and future QoEprovisioning. The exploitation of these methods can be employed for the efficient measurement of other quality related KPIs of a network, as well as, in order to disseminate this information back to the network's subscribers. One potential extension could be the comparison with other geostatistical algorithms for the selection of the optimal one, in correspondence with the KPI under investigation. Moreover, selected user trends could be exploited by different departments of an MNO in order to offer personalized experience to their customers.

\section{ACKNOWLEDGMENTS}

The author would like to thank Lefteris Papakostas, Olga Karali as well as Julian Gyokov from GyokovSolutions and Giannis Nanos from Victus Networks for their fruitful discussions and help that make possible this research.

\section{REFERENCES}

[1] 3GPP, 3rd Generation Partnership Project. Evolved Universal Terrestrial Radio Access (E-UTRA); Study on Minimization of Drive-Tests in Next Generation Networks, 3GPP Standard. Technical Report TS 36.805 v.9.0.0, December 2009.

[2] 3GPP, 3rd Generation Partnership Project. Universal Terrestrial Radio Access (UTRA) and Evolved Universal Terrestrial Radio Access (E-UTRA); Radio Measurement Collection for Minimization of Drive Tests (MDT); Overall Description; Stage 2, 3GPP Standard. Technical Report TS 37.320 v.11.1.0, September 2012.

[3] J. Arpee, S. Gutowski, and M. Touati. Apparatus and method for geostatistical analysis of wireless signal propagation, Mars 2004. US Patent 6,711,404.

[4] Sascha Berger, Albrecht J. Fehske, Paolo Zanier, Ingo Viering, and Gerhard Fettweis. On the advantages of location resolved input data for throughput optimization algorithms in self-organizing wireless networks. In GLOBECOM Workshops, pages 288-292, 2013.

[5] Federico Castanedo. A review of data fusion techniques. The Scientific World Journal, 2013(Article ID 704504):1-19, 2013.

[6] Fedor Chernogorov and Jani Puttonen. User satisfaction classification for minimization of drive tests QoS verification. In Personal Indoor and Mobile Radio Communications (PIMRC), 2013 IEEE 24th International Symposium on, pages 2165-2169, September 2013.

[7] A Faggiani, E. Gregori, L. Lenzini, V. Luconi, and A Vecchio. Smartphone-based crowdsourcing for network monitoring: Opportunities, challenges, and a case study. Communications Magazine, IEEE, 52(1):106-113, January 2014.

[8] A Galindo-Serrano, B. Sayrac, S. Ben Jemaa, J. Riihijarvi, and P. Mahonen. Automated coverage hole detection for cellular networks using radio environment maps. In Modeling Optimization in Mobile, Ad Hoc Wireless Networks (WiOpt), 2013 11th International Symposium on, pages 35-40, May 2013.

[9] A Galindo-Serrano, B. Sayrac, S. Ben Jemaa, J. Riihijarvi, and P. Mahonen. Harvesting MDT data: Radio environment maps for coverage analysis in cellular networks. In Cognitive Radio Oriented Wireless Networks (CROWNCOM), 2013 8th International Conference on, pages 37-42, July 2013.

[10] C Granell, L Díaz, M Gould, V Pascual, J Guimet, P Carrara, and $\mathrm{M}$ Pepe. Developing geoprocessing services for a hydrological model application. In 27th EARSeL Symposium: Geoinformation in Europe, Bolzano, Italy, June 2007.

[11] D.L. Hall and J. Llinas. An introduction to multisensor data fusion. Proceedings of the IEEE, 85(1):6-23, Jan 1997.

[12] W.A Hapsari, A Umesh, M. Iwamura, M. Tomala, B. Gyula, and B. Sebire. Minimization of drive tests solution in 3GPP. Communications Magazine, IEEE, 50(6):28-36, June 2012.

[13] Mikael Jern and Johan Franze?n. Geoanalytics ? exploring spatio-temporal and multivariate data. In Proceedings of the Information Visualization (IV?06), 2006.

[14] J. Johansson, W.A Hapsari, S. Kelley, and G. Bodog. Minimization of drive tests in 3GPP release 11. Communications Magazine, IEEE, 50(11):36-43, November 2012. 
[15] Amila Karunathilake, M. A. P. Chamikara, and Jagath Gunatilake. Web GIS to identify the problematic mobile signal clusters. International Journal of Computer Applications, 88(10):30-34, February 2014.

[16] S. Kolyaie, M. Yaghooti, and M. Gilda. Analysis and simulation of wireless signal propagation applying geostatistical interpolation techniques. Archives of Photogrammetry, Cartography and Remote Sensing, 22:261-270, 2011.

[17] Jin Li and A.D. Heap. A Review of Spatial Interpolation Methods for Environmental Scientists. Record (Australia. Geoscience Australia). Geoscience Australia, 2008.

[18] Georges Matheron. Principles of geostatistics. Economic Geology, 58(8):1246-1266, December 1963.
[19] J. Mitola. Cognitive radio: an integrated agent architecture for software defined radio. $\mathrm{PhD}$ thesis, Stockholm, Sweden, 2000.

[20] W. Muller and H. Schumann. Visualization methods for timedependent data. In Proceedings of the 2003 Winter Simulation Conference, pages 737-746, 2003.

[21] Nokia Siemens Networks et al. Rel-9 Self-Organizing Network (SON). In 3GPP TSG RAN\#43 meeting, number Tdoc RP 090162, Biarritz, France, Mars 2009.

[22] OGC. Open Geospatial Consortium, 2014 (last accessed August 23, 2014).

[23] OGC. Open Geospatial Consortium Web Services, 2014 (last accessed August 23, 2014).

[24] C. Phillips, M. Ton, D. Sicker, and D. Grunwald. Practical radio environment mapping with geostatistics. In Dynamic Spectrum Access Networks (DYSPAN), 2012 IEEE International Symposium on, pages 422-433, October 2012.

[25] Youping Zhao, Bin Le, and Jeffrey H. Reed. Chapter 11 network support: The radio environment map. In Bruce A. Fette, editor, Cognitive Radio Technology, pages 337 - 363 . Newnes, Burlington, 2006. 